\title{
Praxisverkauf
}

\section{Verdruss mit dem Verkehrswert}

\author{
Nicht genug, dass es zunehmend schwer fällt, Nachfolger für die \\ Praxis zu finden. Für den abgebenden Arzt ist auch der vereinbarte \\ Kaufpreis nicht ohne Risiko. Das hat vor kurzem ein Urteil aus \\ München wieder vor Augen geführt.
}

W er den Verkaufspreis seiner Praxis in die Altersvorsorge einkalkuliert hat, sollte vorsichtig sein. Dies musste kürzlich ein Psychotherapeut erfahren, der sich bei der Praxisabgabe mit dem Nachfolger auf eine Summe von 48.000 Euro geeinigt hatte. Das böse Erwachen kam, als der Käufer, nachdem er die Zulassung hatte, die Zahlung verweigerte. Denn letztlich, so das Argument, sei es dem Vorgänger nur um den Verkauf der Zulassung gegangen.

Das Landgericht München (Az.: $23 \mathrm{O}$ 13866/06) ließ den Wert der Praxis daraufhin schätzen. Als der Sachverständige einen Praxiswert von lediglich 9.200 Euro feststellte, entschied das Gericht, der Kaufvertrag sei als „wucherartiges Rechtsgeschäft" sittenwidrig und daher nichtig. Die Bewertung gerade von Psychotherapeuten-Praxen ist seit Jahren ein umstrittenes Thema. „Der Wert von Psychotherapeuten-Praxen ist fast nicht objektiv ermittelbar“, sagt dazu Günther Frielingsdorf, vereidigter Sachverständiger zur Bewertung von Arztpraxen in Köln. Das Problem sei, dass Psychotherapeuten wie keine andere Fachgruppe von der persönlichen Leistung abhängig seien. Ein Patientenstamm ist nicht ohne weiteres übertragbar.

\section{Thema geht auch Fachärzte an}

Die Entscheidungen von Zulassungsausschuss und Landgericht zeigen mit besonderer Deutlichkeit, welche Probleme sich daraus ergeben können, dass der zivilrechtliche Praxiskaufvertrag und die vertragsarztrechtliche Nachfolgezulassung nur partiell miteinander verknüpft sind, und das gilt nicht nur für Psychotherapeuten. Bei Facharztpraxen geht es dabei noch um weit höhere Summen als in dem hier vorgestellten Fall. Das Nachfolgezulassungsverfahren setzt zwar voraus, dass eine veräußerungsfähige Praxis vorliegt und diese auch verkauft werden soll, die Belange des Verkäufers sind in diesem Verfahren aber nur insofern zu berücksichtigen, als der Kaufpreis die Höhe des Verkehrswertes der Praxis nicht übersteigt.

Wenn also ein Nachfolger zugelassen wird und dieser später einwendet, der vertragliche Kaufpreis sei deutlich überhöht und der Vertrag deshalb nichtig, behält er dennoch die vertragsärztliche Zulassung. Der Verkäufer kann diese nicht zurückerhalten. Wie das Urteil des LG München zeigt, kann der Verkäufer bei nichtigem Kaufvertrag auch keinen Schadenersatz oder Wertausgleich für seinen Verzicht auf die Zulassung verlangen. Die Zulassung wird zivil- und vertragsarztrechtlich als nicht handelbares Wirtschaftsgut angesehen. wirtschaftlichen Interessen des verkaufenden Arztes im Nachbesetzungsverfahren nur bis zur Höhe des Verkehrswertes der Praxis berücksichtigt werden. Der Zulassungsausschuss soll nämlich im Sinne der Patientenversorgung den am besten geeigneten Bewerber auswählen, nicht denjenigen, der am meisten bietet.

\section{Praxiswert: Nachfrage zu selten berücksichtigt}

Ob allerdings die Überlegungen des Landgerichts München zur Nichtigkeit des Kaufvertrages ohne Weiteres zutreffen,
Es existieren gute Gründe, warum die

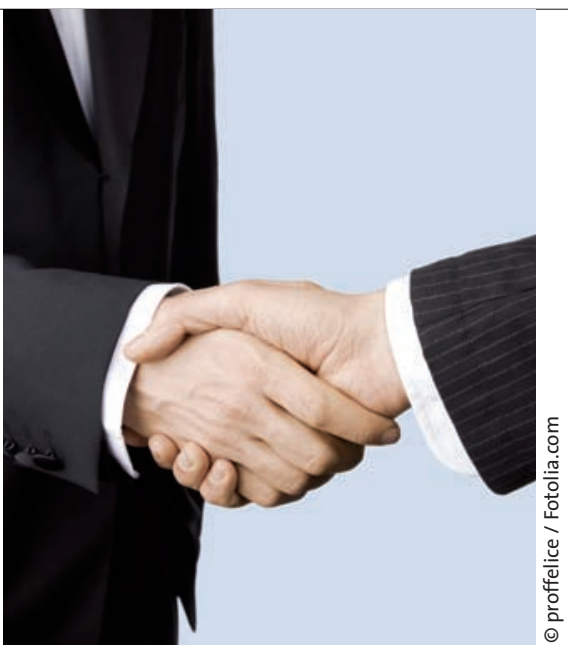

Die Einigung mit dem Praxisnachfolger garantiert nicht, dass der Verkaufspreis auch vor Gericht Bestand hat.

darf bezweifelt werden. Häufig wird bei der Ermittlung des Praxiswerts nämlich nicht berücksichtigt, wie viele Käufer an der Praxis interessiert sind. Dass dieses Interesse auch durch die Möglichkeit der Zulassung zur vertragsärztlichen Versorgung mitbestimmt wird, ist evident und legitim, da die Zulassung den dauerhaften Zugang zu einem reglementierten Markt, nämlich der Versorgung gesetzlich Versicherter, eröffnet. Das zeigt auch die aktuelle Rechtsprechung zur Abschreibung des Praxiskaufs: Der Wert der Zulassung, der eigentlich keine Rolle spielen dürfte, wird von Finanzgerichten als nicht abschreibungsfähig angesehen, weil er ein „nicht abnutzbares Gut" ist. Bei Kaufpreisverhandlungen spielt es sehr wohl eine Rolle, ob nur ein Bewerber oder viele existieren. Im Zusammenhang mit der Praxisveräußerung wird daher - häufig allerdings unausgesprochen - vereinbart, dass sich der Verkäufer im Rahmen seiner Möglichkeiten für die Nachfolgezulassung des Käufers einsetzt.

Eine Möglichkeit, die Probleme mit dem Kaufpreis zu umgehen, könnte es sein, für diese Mitwirkung im Zulassungsverfahren einen zusätzlichen Betrag zu vereinbaren, der neben dem Wert der Praxis abzugelten ist. Dies hat zwar steuerliche Folgen für Käufer und Verkäufer. Bis die Fragen, die durch das LG München aufgeworfen wurden, höchstrichterlich geklärt sind, reduziert dieses Vorgehen aber die Risiken des Verkäufers deutlich.

RA Dr. Ingo Pflugmacher, Bonn Hauke Gerlof, Neu Isenburg 